\section{Case Report \\ Stenotrophomonas maltophilia endogenous endophthalmitis: clinical presentation, sensitivity spectrum and management}

\author{
T. Das, ${ }^{1}$ H. S. Deshmukh, ${ }^{1}$ A. Mathai ${ }^{1}$ and Ashok Kumar Reddy ${ }^{2}$ \\ ${ }^{1}$ Smt Kanuri Santhamma Retina Vitreous Centre, L V Prasad Eye Institute, Hyderabad, India \\ 2Jhaveri Microbiology Centre, L V Prasad Eye Institute, Hyderabad, India
}

Ashok Kumar Reddy

ashokkumar@lvpei.org

Received 16 January 2009

Accepted 3 March 2009

\begin{abstract}
Stenotrophomonas maltophilia is gaining importance as a community-acquired pathogen, after becoming firmly established as a nosocomial pathogen. Here we report a case of endogenous endophthalmitis due to $S$. maltophilia. Antibiotic-susceptibility testing of the isolate was performed by the Kirby-Bauer disc diffusion method. The organism was resistant to aminoglycosides, imipenem, ticarcillin and cotrimoxazole and was sensitive to ceftazidime and chloramphenicol. The patient was successfully treated with a sensitivity-based intravitreal antibiotic regimen.
\end{abstract}

\section{Introduction}

Stenotrophomonas maltophilia is a nonfermentative Gramnegative bacillus previously known as Pseudomonas maltophilia or Xanthomonas maltophilia (Palleroni \& Bradbury, 1993; Swings et al., 1983). The various infections caused by $S$. maltophilia include bacteraemia, pneumonia, urinary tract infection, endocarditis, meningitis, peritonitis and ocular infections (Denton \& Kerr, 1998). Treatment of S. maltophilia infection is often difficult as it is resistant to many antimicrobial agents and antimicrobial resistance may emerge during therapy (Felegie et al., 1979). A variety of ocular infections have been reported with S. maltophilia, including conjunctivitis, keratitis, dacryocystitis, preseptal cellulitis and endophthalmitis (Penland \& Wilhelmus, 1996). Endophthalmitis thus far reported has been following trauma, cataract surgery or intravitreal injection (Benian et al., 2002). Here we report a case of endogenous endophthalmitis due to this organism.

\section{Case report}

A 30-year-old male presented to us with a history of painless acute-onset deterioration of vision in the left eye for 3 days. He had a mild fever 2 weeks previously of 1 day duration which subsided without treatment. The patient had no history of prior hospitalization or intravenous drug use or significant trauma to the eye or elsewhere. He was diagnosed locally as having endogenous endophthalmitis of the left eye and received intravitreal vancomycin, ceftazidime and dexamethasone before being referred to our Centre.

At presentation, the right eye was normal with unaided vision of 6/6, N6. The left eye had a best-corrected visual acuity of perception of light with accurate projection of rays. Anterior segment examination revealed cells $4+$, flare and a $1 \mathrm{~mm}$ hypopyon. There was vitritis with cotton ball exudates in the vitreous. Though the disc was hazily seen, retinal vessels could not be visualized. A clinical impression of left eye endogenous endophthalmitis was formed and pars plana vitrectomy with intravitreal injection of vancomycin and ceftazidime was done.

He was started on oral ciprofloxacin $750 \mathrm{mg}$ b.d. along with topical ciprofloxacin, homatropine and betamethasone. A vitreous sample was sent to Microbiology for culture and for pan-fungal PCR. Direct microscopic examination of the vitreous sample showed Gram-negative bacilli. Confluent growth of Gram-negative bacilli was observed on blood and chocolate agar after $24 \mathrm{~h}$ incubation. The organism was identified as $S$. maltophilia using Mini API ID 32GN strips (bioMérieux). Susceptibility testing was performed by the Kirby-Bauer disc diffusion method. The organism was sensitive to ciprofloxacin, gatifloxacin, moxifloxacin and chloramphenicol and showed intermediate sensitivity to ceftazidime. It was resistant to aminoglycosides, imipenem, ticarcillin and cotrimoxazole. PCR for fungus was negative. Blood and urine cultures were sterile. Routine haemogram, urine examination and chest X-ray were normal. The patient was negative for HIV, the Widal test and the malaria parasite. There was subsequent improvement in visual status to finger counting close to face. The patient continued to show steady improvement in visual status, reaching 20/80p, $\mathrm{N} 12$ on follow-up. At this point, oral ciprofloxacin was stopped and the patient was continued only on topical medications.

A week later, he presented with moderate pain and redness in the left eye of 1 day duration, and vision of finger counting at $2 \mathrm{~m}$. Intraocular pressure was normal 
(14 mmHg). Persistent endophthalmitis of the left eye was diagnosed and an intravitreal injection of chloramphenicol was given on the same day. Oral steroid was added to the existing regimen, and clinical improvement ensued for 1 month when he had a vision of 20/200. He returned 3 weeks later with complaints of pain and redness of the left eye, vision of light perception and accurate projection and was diagnosed with spontaneous lens capsular rupture causing cataract, secondary glaucoma and phacogenic uveitis. Pars plana lensectomy, revitrectomy and intravitreal injection of chloramphenicol, ceftazidime and dexamethasone were done. Repeat blood and urine samples were sterile. Vitreous samples sent this time also showed growth of $S$. maltophilia. Repeat sensitivity profiling showed the organism to be resistant to ceftazidime and intermediately sensitive to ciprofloxacin. The sensitivity pattern remained the same to other antibiotics. The patient's postoperative course stabilized, with the endophthalmitis, phacogenic uveitis and secondary glaucoma resolving and vision improving to 6/30, N12.

\section{Discussion}

S. maltophilia is emerging as an important opportunistic ocular pathogen (Penland \& Wilhelmus, 1996). Endophthalmitis by this organism has been known to occur following trauma, intraocular lens implantation or intravitreal injections (Benian et al., 2002). However, to the best of our knowledge there has been no previous report of the organism causing endogenous endophthalmitis. In our patient, the portal of entry of the organism was uncertain. Extensive probing of history and previous medical records revealed a history of mild self-limited fever of 1 day duration, 2 weeks prior to the development of endophthalmitis. There was no history of hospitalization or any medical intervention in the patient or any trauma in the recent past.

The absence of ocular compromise and medical intervention points towards community-acquired Stenotrophomonas bacteraemia. In our case, there was no growth in the blood and urine cultures on two separate occasions, but the organism was isolated from the vitreous cavity on two separate occasions 2 months apart. This reflects the difficulty in getting positive blood and urine cultures in cases of endogenous bacterial endophthalmitis (Jackson et al., 2003). Whether community-acquired Stenotrophomonas bacteraemia as fleeting and trivial as to cause self-limiting fever of 1 day duration can cause endogenous bacterial endophthalmitis is open to discussion. However, cases of endogenous bacterial endophthalmitis with no or mild systemic symptoms have been reported in the literature. In a recent review, $18 \%$ of endogenous bacterial endophthalmitis patients did not show significant constitutional symptoms (Jackson et al., 2003).
S. maltophilia has several different mechanisms of resistance for different classes of drugs (Crossman et al., 2008). Moreover, it can develop resistance to a drug to which it was previously sensitive (Denton \& Kerr, 1998; Penland \& Wilhelmus, 1996). In our case, the organism showed decreased sensitivity to ceftazidime and ciprofloxacin in a span of 2 months. The accurate identification of this organism is vital as apposed to the mere identification of Gram-negative bacilli. S. maltophilia is characteristically resistant to the traditional antibiotics used against Gramnegative organisms, most notably the aminoglycosides (Denton \& Kerr, 1998). In our case, however, the organism was sensitive to chloramphenicol on two separate occasions, even though it was resistant to cotrimoxazole, which has often been mentioned as the drug of choice (Al-Jasser, 2006). Its repeated in vitro sensitivity to chloramphenicol and excellent clinical response opened up an effective and relatively nontoxic line of treatment. To summarize, $S$. maltophilia can cause endogenous endophthalmitis, which can be treated successfully and useful vision salvaged with antibiotic sensitivity-guided antibacterial therapy.

\section{Acknowledgements}

We acknowledge the Hyderabad Eye Research Foundation for funding.

\section{References}

Al-Jasser, A. M. (2006). Stenotrophomonas maltophilia resistant to trimethoprim-sulfamethoxazole: an increasing problem. Ann Clin Microbiol Antimicrob 5, 23.

Benian, O., Alimgil, L. \& Erda, N. (2002). Two cases of Stenotrophomonas maltophilia endophthalmitis. Ophthalmic Surg Lasers 33, 253-256.

Crossman, L. C., Gould, V. C., Dow, J. M., Vernikos, G. S., Okazaki, A., Sebaihia, M., Saunders, D., Arrowsmith, C., Carver, T. \& other authors (2008). The complete genome, comparative and functional analysis of Stenotrophomonas maltophilia reveals an organism heavily shielded by drug resistance determinants. Genome Biol 9, R74.

Denton, M. \& Kerr, K. G. (1998). Microbiological and clinical aspects of infection associated with Stenotrophomonas maltophilia. Clin Microbiol Rev 11, 57-80.

Felegie, T. P., Yu, V. L., Rumans, L. W. \& Yee, R. B. (1979). Susceptibility of Pseudomonas maltophilia to antimicrobial agents, singly and in combination. Antimicrob Agents Chemother 16, 833-837.

Jackson, T. L., Eykyn, S. J., Graham, E. M. \& Stanford, M. R. (2003). Endogenous bacterial endophthalmitis: a 17 -year prospective series and review of 267 reported cases. Surv Ophthalmol 48, 403-423.

Palleroni, N. J. \& Bradbury, J. F. (1993). Stenotrophomonas, a new bacterial genus for Xanthomonas maltophilia. Int J Syst Bacteriol 43, 606-609.

Penland, R. L. \& Wilhelmus, K. R. (1996). Stenotrophomonas maltophilia ocular infections. Arch Ophthalmol 114, 433-436.

Swings, J., De Vos, P., Van den Mooter, M. \& De Ley, J. (1983). Transfer of Pseudomonas maltophilia Hugh 1981 to the genus Xanthomonas as Xanthomonas maltophilia (Hugh 1981) comb nov. Int J Syst Bacteriol 33, 409-413. 